\title{
Experimental Study on the Starting-up and Heat Transfer Characteristics of Pulsating Heat Pipe under Local Low-frequency Vibrations Jing Chen', Junbiao Dong ${ }^{2}$, Ye Yao ${ }^{2 *}$
}

1. Nanjing Vocational lnstitute of Transport Technology, Nanjing, Jiangsu, 211188, China

2. Institute of Refrigeration and Cryogenics, Shanghai Jiao Tong University, Shanghai, 200240, China

\begin{abstract}
:
This study mainly experimentally investigates and explores the effects of local low-frequency vibrations on the starting-up and heat transfer characteristics of the pulsating heat pipe. A micro motors with the vibration frequency of $200 \mathrm{~Hz}$ were imposed on the external surface of evaporation, condensation and adiabatic section of the pulsating heat pipe, respectively, and the starting-up temperature and the average temperatures along the evaporation section as well as the thermal performances of the vibrating heat pipe were experimentally scrutinized under the local vibrations of different positions. The following important conclusions can be achieved by the experimental study: 1) The effect of vibrations at the evaporation section and at the adiabatic section on the starting-up time of pulsating heat pipe is more significant than that at the condensation section. 2) The vibrations at different positions can reduce the starting-up temperature of the pulsating heat pipe. The effect of the vibrations at the evaporation section is the best as the heating power is lower, and the effect of the vibration at the adiabatic section is the best as the heating power is higher. 3) The vibrations at the evaporation section and at the adiabatic section can reduce the thermal resistance of the pulsating heat pipe. However, the vibrations at the condensation section have little effect on the thermal resistance of the pulsating heat pipe. 4) The vibrations at the evaporation section and at the adiabatic section can effectively reduce the temperature of evaporation section of the pulsating heat pipe, but the vibrations at the condensation section have no effect on the temperature of evaporation section of the pulsating heat pipe.
\end{abstract}

Keywords : pulsating heat pipe; local vibration; starting-up characteristic; heat transfer performance

\section{Introduction}

Heat pipe, also known as "thermal superconducting tube", is a heat transfer technology developed

Corresponding Author: Ye Yao, yeyao10000@sjtu.edu.cn 
in the 1960s. It adopts vaporization and condensation phase transformation of its internal saturated working medium to realize heat transfer. This phase transformation heat transfer mode has a very high heat transfer capacity. Compared with copper, aluminum and other metals with good heat conductivity, the heat transfer capacity of heat pipe is several orders of magnitude higher [1]. In addition to its excellent heat transfer, heat pipe has many other outstanding advantages such as good isothermal property, heat flux density variability, flow direction reversibility and environmental self-adaptability, and hence, it has been widely used in the field of heat dissipation of electronic equipments, aerospace and military industry [2].

As a new type of heat dissipation device proposed by Akachi in the 1990s [3], pulsating heat pipe has been increasingly focused by people due to its advantages of simple structure, small volume, reliable operation, flexible arrangement and high heat transfer efficiency compared with traditional heat pipes. The working principle of pulsating heat pipe is illustrated as below: liquid working medium (distilled water, methanol, acetone, freon, etc.) in serpentine capillary tube forms many liquid column and gas plug with different lengths under the action of surface tension, and the gas and liquid plugs create an unstable, random pulsating flow between the evaporation and cooling sections due to the thermally heating force, i.e. it oscillates back and forth between evaporation and condensation to achieve efficient heat transfer.

Although the structure of pulsating heat pipe is simple, the gas-liquid two-phase flow inside the heat pipe involves many heat transfer mechanisms, such as sensible heat transfer, latent heat transfer and expansion work. A large number of studies have shown that the working medium, working conditions and geometric parameters of pulsating heat pipe produce important effects on its startingup and heat transfer characteristics. Kangli Bao et al. [4] and A. Gandomkar et al. [5] studied the effects of surfactants on the starting-up, operation and drying characteristics of pulsating heat pipes. The results showed that surfactants could reduce the thermal resistance and starting-up thermal load and increase the heat load of burning and drying. To solve the difficulty of starting pulsating heat pipes at low heating power, Zhang et al. [6] proposed a zeotropic immiscible mixture (HFE-7100) as the working fluid. The results showed that compared with water, the low latent heat of vaporization of HFE-7100 could accelerate the formation of bubbles in evaporation section and the starting-up of the pulsating heat pipes. In terms of working conditions, Ahmad et al. [7] investigated the influence of 
filling ratios of working fluid (ethanol) on the thermal performance of the pulsating heat pipes. The experimental results indicated that the optimal filling ratio was $50 \%$, and the thermal resistance significantly decreased with an increase in the heat input power for a filling ratio of 50\% ethanol. Bai et al. [8] explored effect of vacuum on heat transfer performance of pulsating heat pipe charged with de-ionized water or $\mathrm{A} 12 \mathrm{O} 3$ /water nanofluid. Experimental results showed that vacuum degree had an important effect on the starting-up and heat transfer performance of pulsating heat pipe as heat load is more than $90 \mathrm{~W}$. Plus, the starting-up heat load decreased with the increasing of internal vacuum degree of the pulsating heat pipe. Huang et al. [9] introduced a gas-liquid two-phase flow model into two-gas plug-one liquid plug heat transfer unit model to study the influence of different fluid states of the pulsating heat pipe on its heat transfer performance. The results showed that the improved model could better reflect the change of heat transfer performance of heat pipes under different heat load conditions. Other researchers improved the heat transfer performances through changing the structure form. For example, Bao et al. [10] proposed a metal foam multichannel heat pipe with the distilled water as the working fluid and studied its heat transfer characteristics. Compared with the traditional pulsating heat pipe, the metal foam multichannel heat pipe showed the better heat transfer performance and the better stability and uniformity of the evaporation temperature. Jiang et al. [11] proposed a novel saw-tooth corrugated structure pulsating heat pipe with two-bends which has been proved to have much better heat transfer performance and shorter starting-up time than the pulsating heat pipe with traditional structure.

Since the heat pipe has complex gas-liquid two phase pulsating flow, the stability and heat transfer performance of its internal working medium will be affected by external vibration to some extent. Therefore, it is necessary to fully understand the various properties and heat transfer mechanism of heat pipe under vibration environment through experimental study. Alaei et al. [12] studied the effects of low-frequency vibrations on a horizontal heat pipe, and it was found that the low-frequency vibrations imposed a significant effect on the thermal performance and the best performance was achieved with the thermal resistance $0.05 \mathrm{~K} / \mathrm{W}$ in the frequency $25 \mathrm{~Hz}$. In another study by Alaei et al. [13], the effects of low-frequency vibrations on a vertical heat pipe was experimentally investigated under different heat transfer rates, filling ratios and frequencies, and the best performance was achieved with the thermal resistance $0.064 \mathrm{~K} / \mathrm{W}$ in the frequency $30 \mathrm{~Hz}$. Similarly, the low-frequency 
vibrations have been experimentally proved to produce positive influence on the thermal performance of a pulsating heat pipe due to the fact that the low-frequency vibrations could removed the 'dry-out' of the pulsating heat pipe[14]. Chen et al. [15] studied the influence of horizontal longitudinal vibrations on the heat transfer performance of a grooved cylindrical copper heat pipe with a length of $600 \mathrm{~mm}$ and an outer diameter of $8 \mathrm{~mm}$. The results showed that the horizontal vibration in the longitudinal direction caused an increase in the heat transfer of the heat pipe that was directly proportional to the input vibration energy. Guo et al. [16] investigated the effects of vertical mechanical vibration on the flow characteristics of a liquid film in vertical rectangular microgrooves. The vibration frequencies were $6 \mathrm{~Hz}, 10 \mathrm{~Hz}$ and $30 \mathrm{~Hz}$, respectively, and the maximal displacement of the microgroove plate was $4.15 \mathrm{~mm}$. The experimental results showed that the dry point was impelled upward and the wetting length was elongated by the vibration, which might be the reason of heat transfer enhancement brought by the vibrations. Zhang et al. [17] investigated the effect of vibration on forced convection heat transfer for $\mathrm{SiO} 2-$ Water nanofluids in a straight circular pipe. Experimental results manifested that the heat transfer coefficient could be enhanced by imposing a transverse vibration on the heat transfer surface (the largest increase of about $182 \%$ was observed). Chen et al. [18] investigated the potential seismic vibrations effect on two-phase flow in an annular channel. In their study, a low-speed eccentric-cam vibration module capable of operating at motor speed of 45$1200 \mathrm{rpm}(\mathrm{f}=0.75-20 \mathrm{~Hz})$ was attached to an annular channel with inner and outer diameters of $19.1 \mathrm{~mm}$ and $38.1 \mathrm{~mm}$, respectively. The void fraction was found to potentially decrease under vibrations in bubbly flow regime and relatively lower liquid flow conditions and increase at the region closed to bubbly-to-slug transition boundary under vibrations, and this could be the essential reason for the heat transfer enhancement by vibrations. Zhou [19] explored the effect of vibration on the heat transfer performance of pulsating heat pipes with different pipe diameters. The results showed that the effect of vibration on the pulsating heat pipe increased with the increase of the pipe diameter.

Known from the above literature, vibration has a significant influence on the operation of pulsating heat pipe. However, the current studies are mainly focused on the overall vibration (i.e., transverse or verticall) and its frequency as well as displacement. There lack of researches about the influence of local vibration and its vibration position on the starting-up and heat transfer characteristics of pulsating heat pipe. Therefore, the purpose of the current study is to investigate and explore the 
effects of local low-frequency vibrations on the thermal performance of the pulsating heat pipe as a novel experimental apparatus and method designed using micro motors. The micro motors with the vibration frequency of $200 \mathrm{~Hz}$ were imposed on the external surface of evaporation, condensation and adiabatic section of the pulsating heat pipe, respectively.The starting-up temperature and the temperatures along the evaporation as well as the thermal performances of the vibrating heat pipe were experimentally scrutinized under the local vibrations of different positions.

\section{Analysis methods}

\subsection{Starting-up characteristics}

The pulsating heat pipe can be deemed to start up when the surface temperature of heat pipe begins to appear peak-and-trough oscillations. Meanwhile, the starting-up of the pulsating heat pipe can be also confirmed by using the distribution theory of statistical random variables, i.e., as long as the heat pipe's temperature exceeds $\left[\mathrm{t}_{0}-3 \sigma, \mathrm{t}_{0}+3 \sigma\right]$ (where $\sigma$ is the standard deviation of the random variable), the pulsating heat pipe can be considered to be started.

(1) Starting-up time

The starting-up of pulsating heat pipe is a random quasi-steady state process. Before starting, the pulsating heat pipe needs a certain amount of time to accumulate heat and increase the temperature of the working medium in the pipe until the starting condition is reached. The time elapsed from the beginning of the input heat load to the starting-up of the pulsating heat pipe is defined as the startingup time of the pulsating heat pipe.

(2) Starting-up temperature

Before the pulsating heat pipe starts, the working medium is in a state of equilibrium. During the starting process, the working medium needs to accumulate a large amount of heat until a certain temperature is arrived at. During the experiment, the temperature of evaporation section required for the starting-up of the pulsating heat pipe is defined as the starting-up temperature of the pulsating heat pipe.

(3) Starting-up power

One of the driving forces of the working fluid in pulsating heat pipe is the pressure difference caused by the bubble expansion due to being heated in the evaporation section and the contraction due 
to being cooled in the condensation section. The growth process of the bubble needs to absorb a large amount of heat in the evaporation section. So, there should be a minimum thermal power that can generate enough pressure difference to promote the flow of the working medium. The minimum power for starting the pulsating heat pipe is the starting-up power of the pulsating heat pipe.

\subsection{Thermal performance evaluation index}

Thermal performances of the pulsating heat pipe mainly include thermal resistance and equivalent thermal conductivity.

Thermal resistance $(\mathrm{R})$ of the pulsating heat pipe mainly consists of heat conduction resistance of the heat pipe wall, phase change heat transfer resistance and convection heat transfer resistance. It can be calculated by Eq.(1)

$$
R=\frac{\bar{t}_{c}-\bar{t}_{e}}{P}
$$

where, $\mathrm{R}$ is total heat transfer resistance, $\mathrm{K} / \mathrm{W} ; \mathrm{P}$ is heating load in the evaporation section, $\mathrm{W} ; \overline{\mathrm{t}}_{c}$ and $\overline{\mathrm{t}}_{\mathrm{e}}$ are average temperature in the condensation and evaporation section, respectively, ${ }^{\circ} \mathrm{C}$.

Equivalent thermal conductivity of the pulsating heat pipe $\left(\lambda_{\text {equ }}\right)$ can be calculated by Eq.(2)

$$
\lambda_{\text {equ }}=Q_{e} \cdot L_{\text {total }} /\left[A\left(\bar{t}_{e}-\bar{t}_{c}\right)\right]
$$

where, $Q_{e}$ is heating load, W; $L_{\text {total }}$ is distance between the center position of the evaporation section and the center position of the condensation section, $\mathrm{m}$; $\mathrm{A}$ is cross-sectional area, $\mathrm{m}^{2}$.

\section{Experimental system}

A pulsating heat pipe has been made by quartz glass tube. Detailed dimensions of the pulsating heat pipe is shown in Tab.1. Miniature vibrating motors (rated voltage: $3.0 \mathrm{~V}$; vibration frequency: 200 $\mathrm{Hz}$ ) were installed on different sections of the pulsating heat pipe (i.e., the evaporation section, condensation section and adiabatic section) to investigate the influences of local vibrations on the working performances of the pulsating heat pipe. The detailed positions of temperature sensors and miniature vibrating motors on the pulsating heat pipe for the experimental study are shown in Fig.1. The working medium of the pulsating heat pipe is deionized water, and the filling rate is $45 \%$. An 
enamelled Ni-Cr resistance wire was used for heating the evaporation section, and the heating power was controlled by A DC voltage and current stabilized power supply. The product of the input voltage and the input current of a DC regulated supply is considered as heating power of pulsating heat pipe. The measurement accuracy of voltage and current is $0.1 \mathrm{~V}$ and $0.01 \mathrm{~A}$, respectively. In order to reduce the heat loss caused by convection and radiation, the heating part was wrapped with thermal insulation film. The condensation section was cooled by an axial flow fan. The experimental setup for testing the thermal characteristics of the pulsating heat pipe is shown in Fig.2.

Table 1 Detailed dimensions of pulsating heat pipe

\begin{tabular}{|c|c|}
\hline Inner diameter $(\mathrm{m})$ & 0.002 \\
\hline Outer diameter $(\mathrm{m})$ & 0.006 \\
\hline Number of elbow & 5 \\
\hline Elbow spacing $(\mathrm{m})$ & 0.06 \\
\hline Elbow outside diameter (m) & 0.036 \\
\hline Evaporation length (m) & 0.1 \\
\hline Condensation length (m) & 0.15 \\
\hline Adiabatic length (m) & 0.05 \\
\hline
\end{tabular}

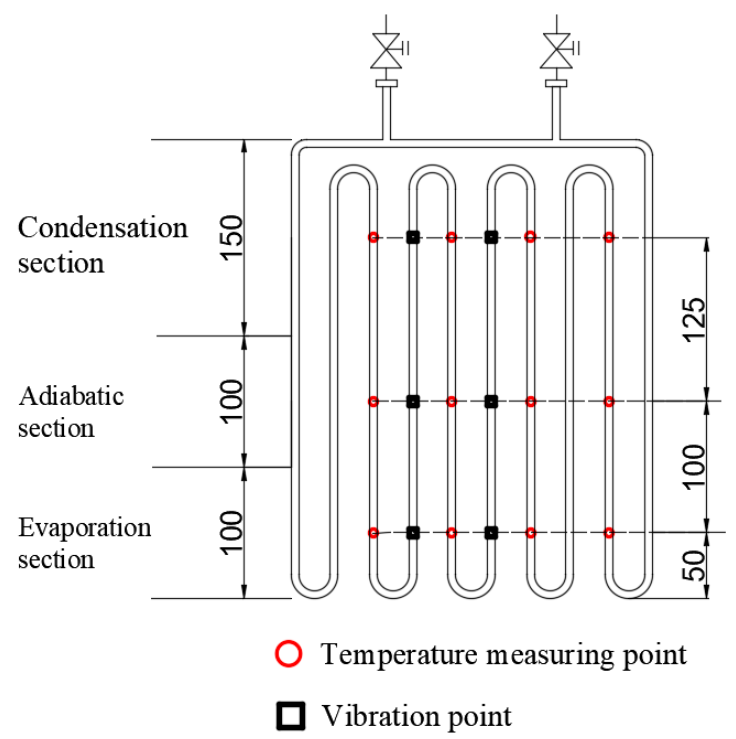

Fig.1 Positions of temperature sensors and miniature vibrating motors on the pulsating heat pipe for the experimental study 

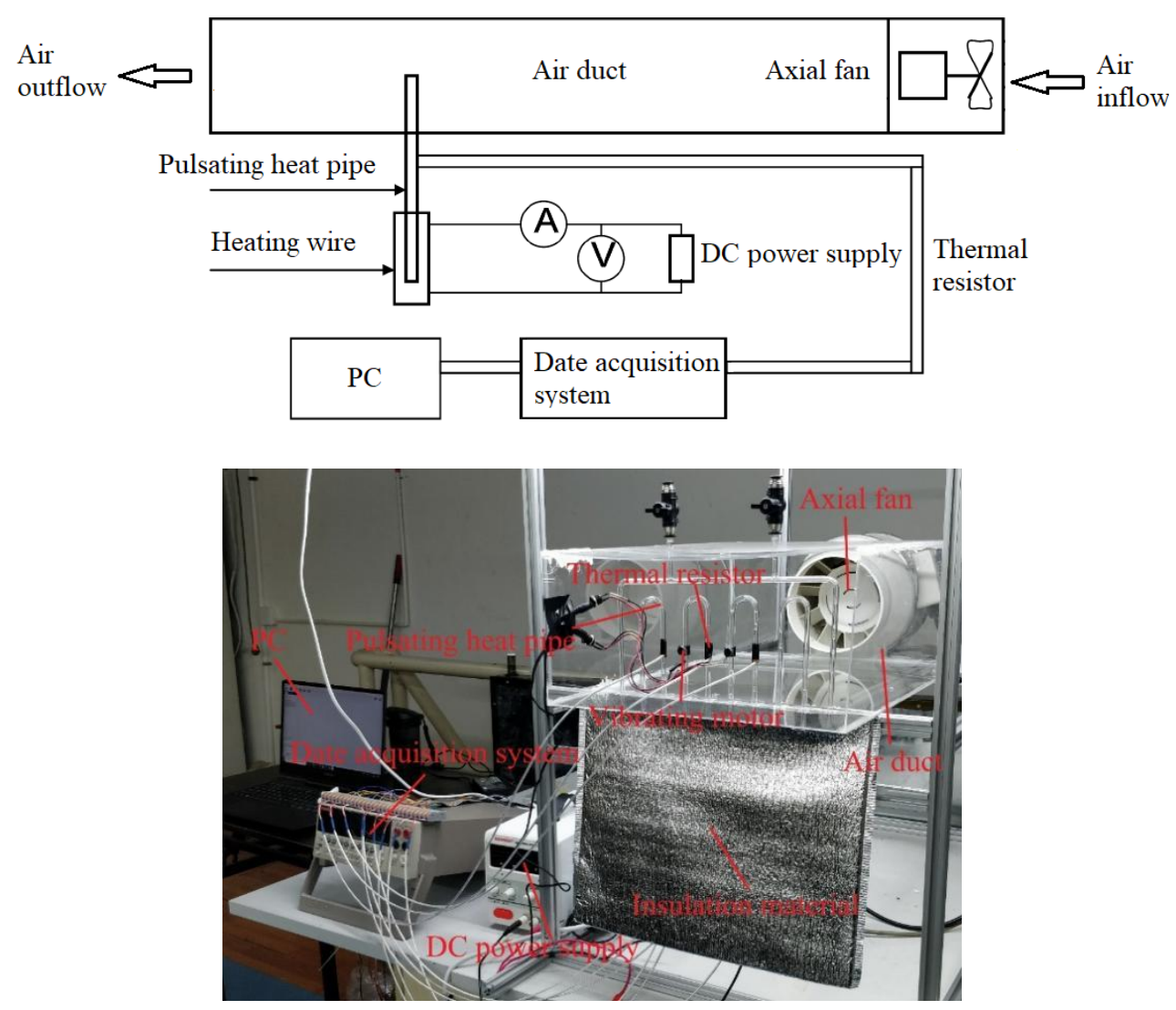

Fig.2 Experimental setup for thermal characteristics test of the pulsating heat pipe

\section{Results and discussion}

\subsection{Influence of vibrations on temperature characteristics of pulsating heat pipe}

Fig.3 shows temperature curves at evaporation section of pulsating heat pipe under different vibration positions (i.e., evaporation section, adiabatic section and condensation section) in the case of heating power of $40 \mathrm{~W}$. As shown in Fig.3, in the preheating process, the four temperature curves basically overlap. This is because most of the heat during the preheating process comes from heating wire, and the heat generated by vibration is small and negligible. With the time going on, the pulsating heat pipe under the vibration condition starts first, while the pulsating heat pipe under the nonvibration condition starts later, which manifests that the vibration shortens the preheating time of the pulsating heat pipe and accelerates the start. This is because when the heating time is short, the pressure difference accumulated by boiling of the working medium in the evaporation section cannot push the liquid plug in the tube to the condensing section, and the pulsating heat pipe cannot be started. The presence of vibration at any sections of the pulsating heat pipe can destroy the boundary layer of the working medium on the wall of the tube, strengthen the convective heat transfer between the working 
medium and the wall of the tube, and the vibration can also accelerate the escape of the bubbles generated by the boiling of the working medium and accelerate the accumulation rate of the pressure difference in the gas plug. It can be seen from Fig. 3 that the starting-up temperature in the case of no vibration is the highest, followed by that in the case of evaporation and adiabatic vibration, and that in the case of condensation vibration is the lowest. This is because vibration can destroy the boundary layer of the working medium on the pipe wall and reduce the resistance of the liquid plug to move from the evaporation section to the condensation section, so it can be started at a lower evaporation temperature. Vibration at condensation section can also enhance the convective heat transfer between the gas or liquid plug and the condensate section tube wall, speeding up the cooling rate, and thus the lower evaporation temperature required for the start.

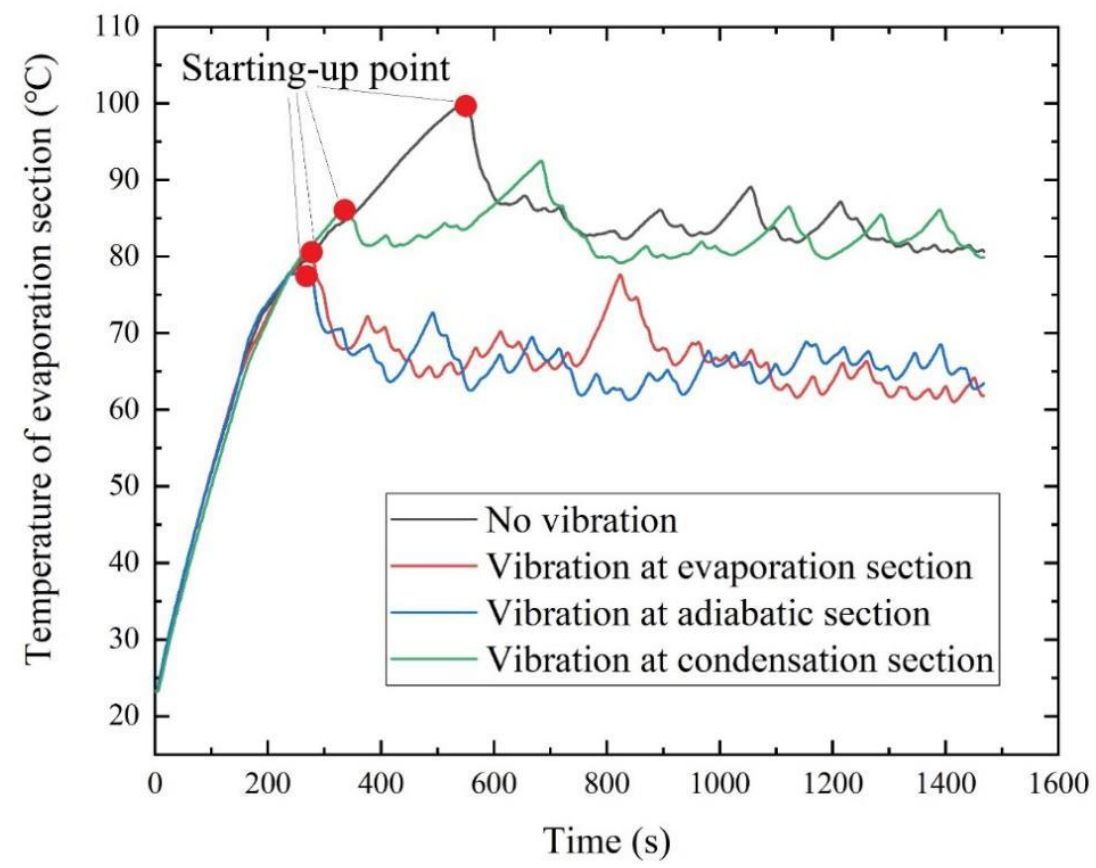

Fig.3 Temperature characteristics at evaporation section of pulsating heat pipe under different vibration positions (Heating power $40 \mathrm{~W}$ )

Fig.4 presents influence of vibrations of different positions on the temperature of evaporation section of pulsating heat pipe under different heating power levels. It can be seen from Fig.4 that with the heating power increasing, the temperature of evaporation section first increases, then basically remains unchanged, and then continues to increase. The phenomenon can be explained as below: when the heating power is low, the thermal resistance of the pulsating heat pipe is large and the heat transfer performance is poor and the pulsating heat pipe can not effectively discharge heat, resulting in the 
temperature rise of the evaporation section with the increase of heating power. With the heating power further increasing, the thermal resistance of the pulsating heat pipe decreases and the heat transfer performance improves, and hence the temperature of the evaporation section does not increase any more. However, as the heating power continues to increase to a certain value, the decrease of the thermal resistance of the pulsating heat pipe is not obvious, so the temperature of the evaporation section begins to rise once again. Comparing the temperature of the evaporation section under different vibration positions, it can be found that the vibrations at the evaporation section and at the adiabatic section can effectively reduce the temperature of the evaporation section under the lower heating power level. For example, when the heating power is $40 \mathrm{~W}$, the vibration has the best effect on reducing the temperature of the evaporation section. In such case, the temperature of the evaporation section can be reduced from $81{ }^{\circ} \mathrm{C}$ to $62{ }^{\circ} \mathrm{C}$ after the vibrations are introduced. However, with the heating power increasing, the effect of vibrations on the temperature of the evaporation section become weakened until it disappears.

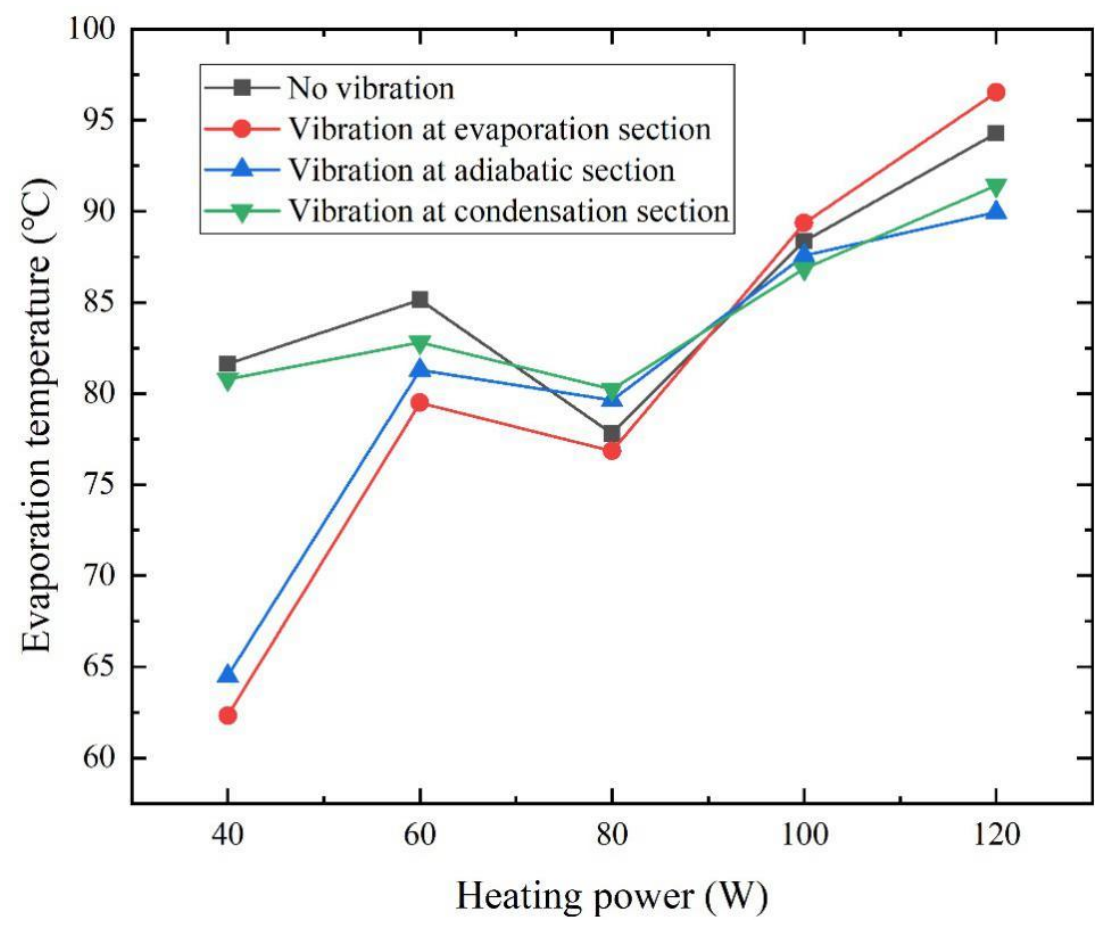

Fig.4 Influence of vibrations of different positions on the temperature of evaporation section of pulsating heat pipe under different heating power levels

\subsection{Influence of vibrations on the starting-up temperature}

In order to further analyze the impact of vibration on the starting-up temperature of the pulsating heat pipe and to compare the impact of different vibration positions, the starting-up temperatures of 
the pulsating heat pipe at the heating power of 40,60, 80, 100 and $120 \mathrm{~W}$ were measured under no vibration and vibration at different positions, i.e, evaporation section, adiabatic section and condensing section. The results are plotted in Fig.5. As shown in Fig.5, in the case of no vibration, the starting temperature decreases first and then increases with the increase of heating power. The phenomenon can be explained by the reason as below: when the heating power is low, the pulsating heat pipe needs a long time to start until the evaporation section is heated to a high temperature. With the increase of heating power, the starting-up time of the pulsating heat pipe becomes shorter and shorter, and the starting-up evaporation temperature is reduced. When the heating power becomes larger, the pulsating heat pipe can quickly start, and the effect of heating power on the starting-up time becomes smaller. At the same time, the increase of heating power would result in the higher evaporation temperature rate. As a result, the evaporation temperature will increase as the pulsating heat pipe is starting.

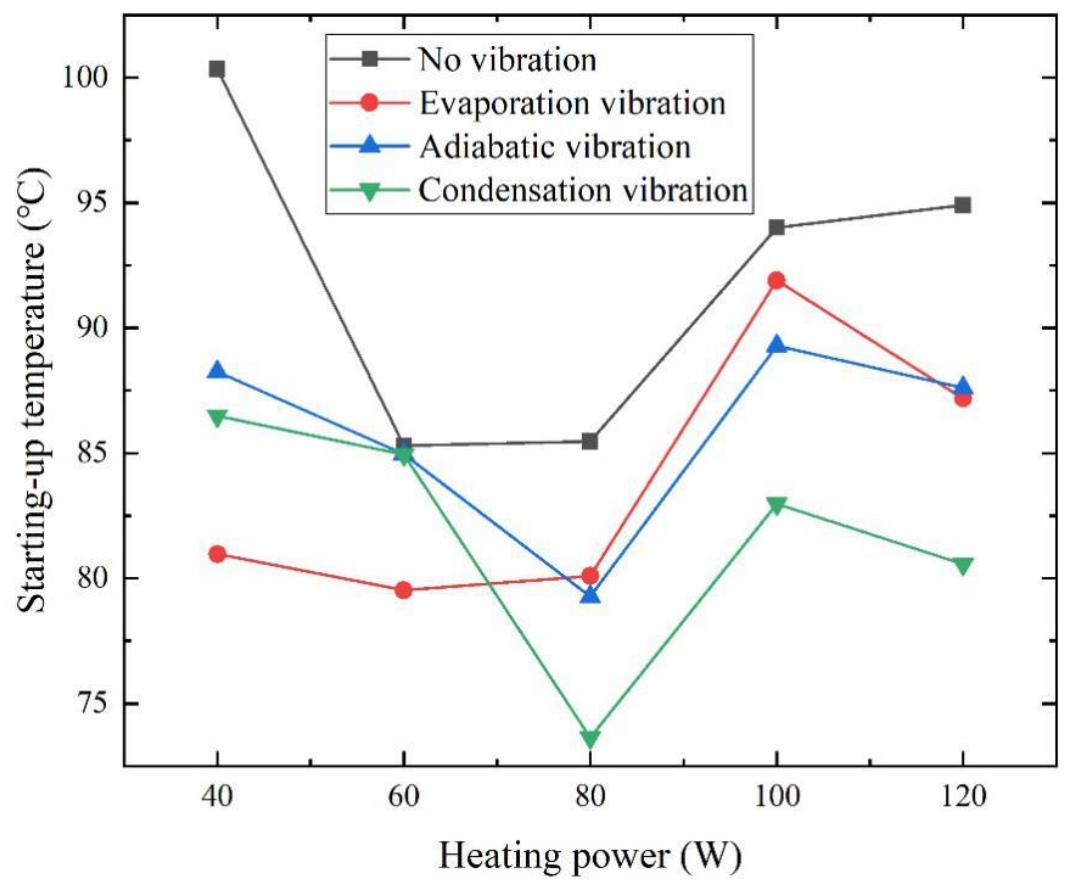

Fig.5 Influence of vibrations of different positions on the starting-up temperature of pulsating heat pipe under different heating power levels

By comparing the starting-up temperature of different vibration positions under the same heating power, it can be found that the starting-up temperature of the three vibration positions is lower than that of the non-vibration conditions, indicating that the vibration at three different positions can reduce the starting-up temperature of the pulsating heat pipe. When the heating power is low, the vibration at the condensing section will reduce the starting-up temperature more effectively. When the heating 
power is high, the vibration at the evaporation section and the adiabatic section will achieve the better effect in the reduction of starting-up temperature.

\subsection{Influence of vibrations on the starting-up time}

Fig.6 presents influence of vibrations on the starting-up time of pulsating heat pipe under different heating power levels $(40 \mathrm{~W}, 60 \mathrm{~W}, 80 \mathrm{~W}, 100 \mathrm{~W}, 120 \mathrm{~W})$. As shown in Fig.6, the starting time decreases with the increase of heating power. This is because the higher the heating power is, the faster the bubbles are generated in the evaporation section, and the pressure difference between the evaporation section and the condensation section can reach the critical pressure difference of startup more quickly. Meanwhile, it can be seen from Fig.6 that the decreasing speed of starting time decreases with the increase of heating power, until the final starting time basically does not change with the increase of heating power. This is because the pulsating heat pipe is difficult to start when the heating power is low, and the starting time can be significantly reduced by increasing the heating power. However, when the heating power is large, the pulsating heat pipe can start quickly. Through comparing the starting time of different vibration conditions under the same heating power, it can be found that the starting time of the three vibration conditions is smaller than that of the no-vibration condition under all heating power, indicating that vibration can effectively reduce the starting time.

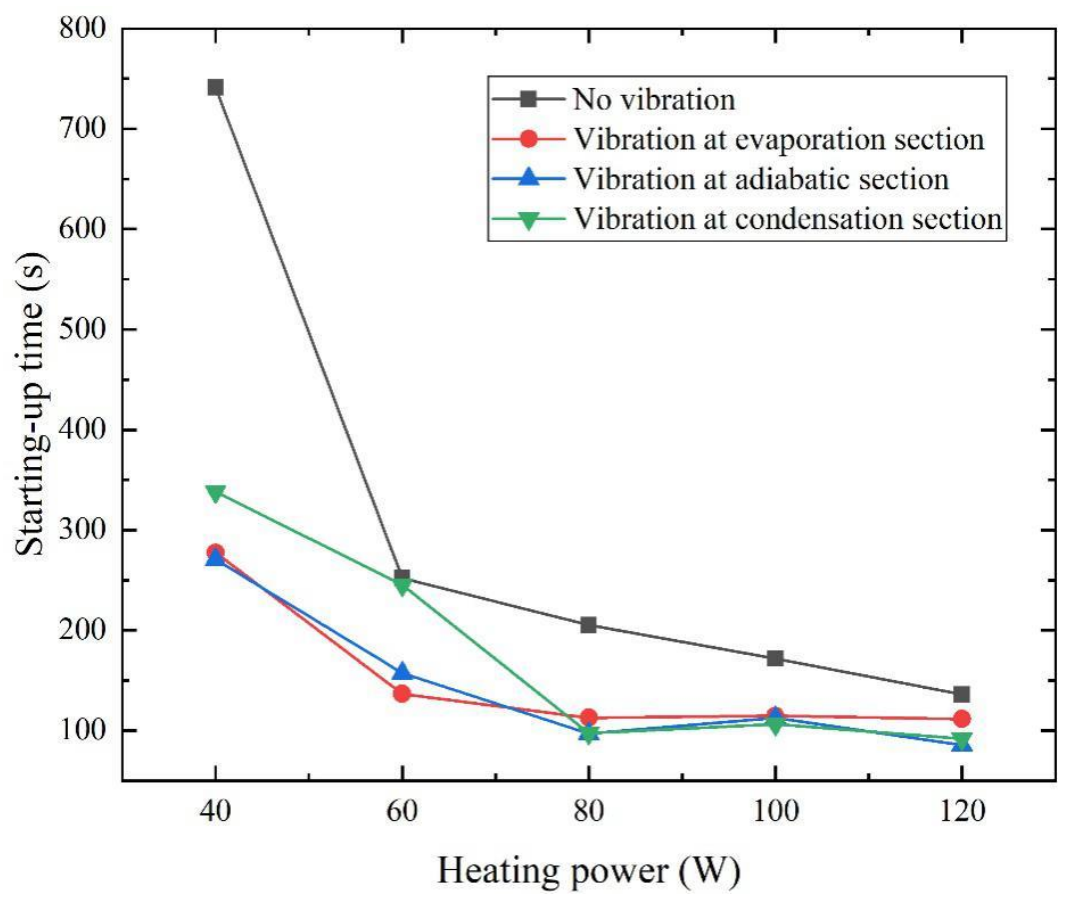

Fig.6 Influence of vibrations on the starting-up time of pulsating heat pipe under different heating power levels 
From Fig,6, we can also see that when the heating power is low, vibration has a significant effect on the reduction of the starting time. However, with the increase of the heating power, the reduction effect becomes worse and worse. This is because when the heating power is small, the pulsating heat pipe is difficult to start, then the external vibration can greatly reduce the start time, but when the heating power is large, the pulsating heat pipe can start faster, and the effect of vibration is not obvious. In this study, vibration has the best effect on reducing the starting time in the case of $40 \mathrm{~W}$ in the heating power when the starting time of the pulsating heat pipe can reduce by about $60 \%$ 。

\subsection{Influence of vibrations on thermal characteristics}

Fig.7 shows influence of vibrations of different positions on the thermal resistance of pulsating heat pipe under different heating power levels (i.e., 40, 60, 80, 100 and $120 \mathrm{~W}$ ). It can be seen from Fig. 6 that under the thermal resistance of pulsating heat pipe decreases with the increase of heating power, and the decreasing slope of thermal resistance decreases with the increase of heating power. This is because with the increase of heating power, the bubble formation rate of evaporation section is accelerated, and the gas plug and liquid plug move faster to the condensation section, which makes the heat transfer performance better. Comparing the thermal resistance of different vibration positions under the same heating power, it can be found that the vibrations at the evaporation section and at the adiabatic section will bring about significant reduction of thermal resistance of the pulsating heat pipe, while the vibrations at the condensation section produce little influence on the thermal resistance of the pulsating heat pipe. The reason is that the vibrations at the evaporation section can be conducive to the bubble separation and enhance the convective heat transfer between working medium and pipe wall so as to accelerate the bubble formation rate. The vibrations at the adiabatic section can enhance the convective heat transfer between working medium and pipe wall, and this improves the heat transfer performance of the pulsating heat pipe. Although the vibrations at the condensation section can also strengthen the convective heat transfer between working medium and pipe wall, the thermal resistance at the condensation section itself is much smaller than that at the evaporation section and at the adiabatic section, which results in the phenomenon that the influence of the vibrations at the condensation section on the thermal resistance of the pulsating heat pipe is relatively small. The results in Fig.7 also manifest that the influence of vibrations on the thermal resistance of the pulsating heat 
pipe becomes increasingly smaller with the increase of heating power level. This is because when the heating power is high, the bubble formation rate in the evaporation section will be very fast, and the enhancement effect of vibrations is not obvious.

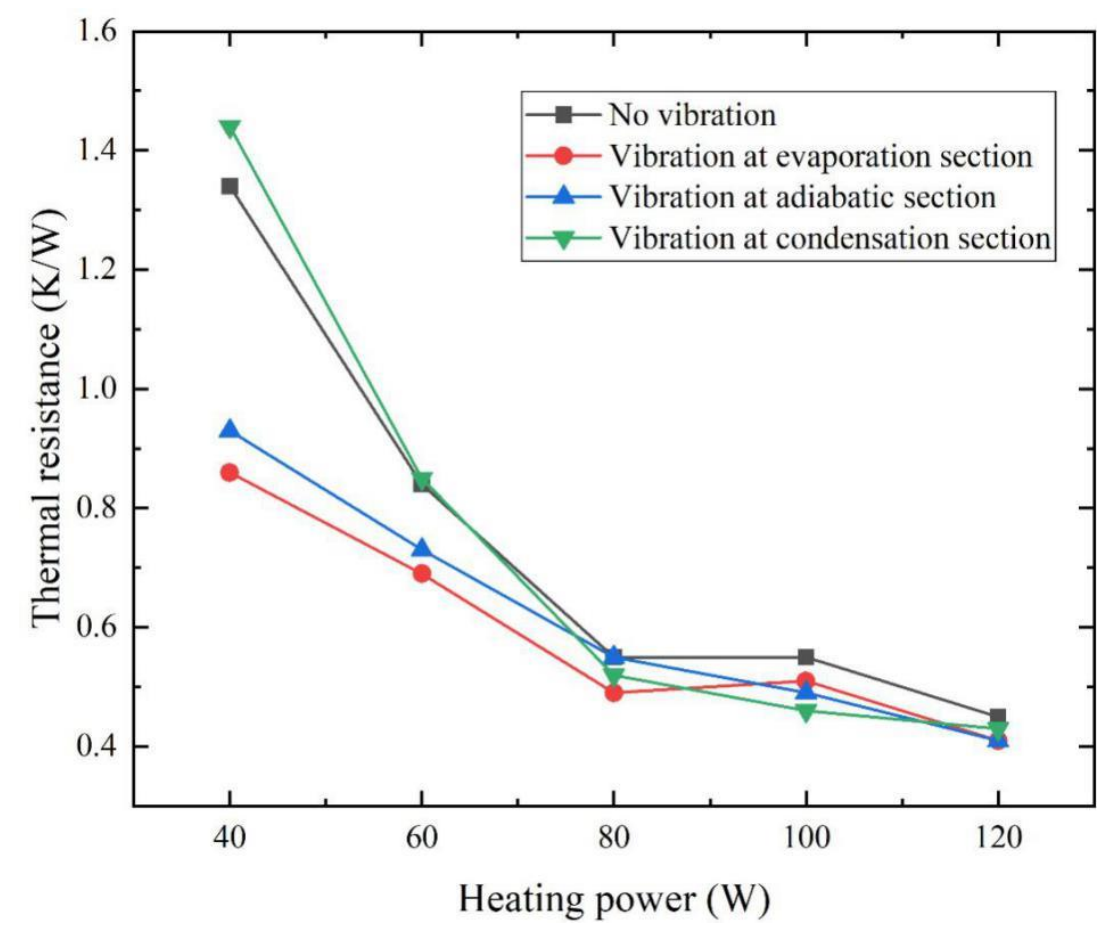

Fig.7 Influence of vibrations of different positions on the thermal resistance of pulsating heat pipe under different heating power levels

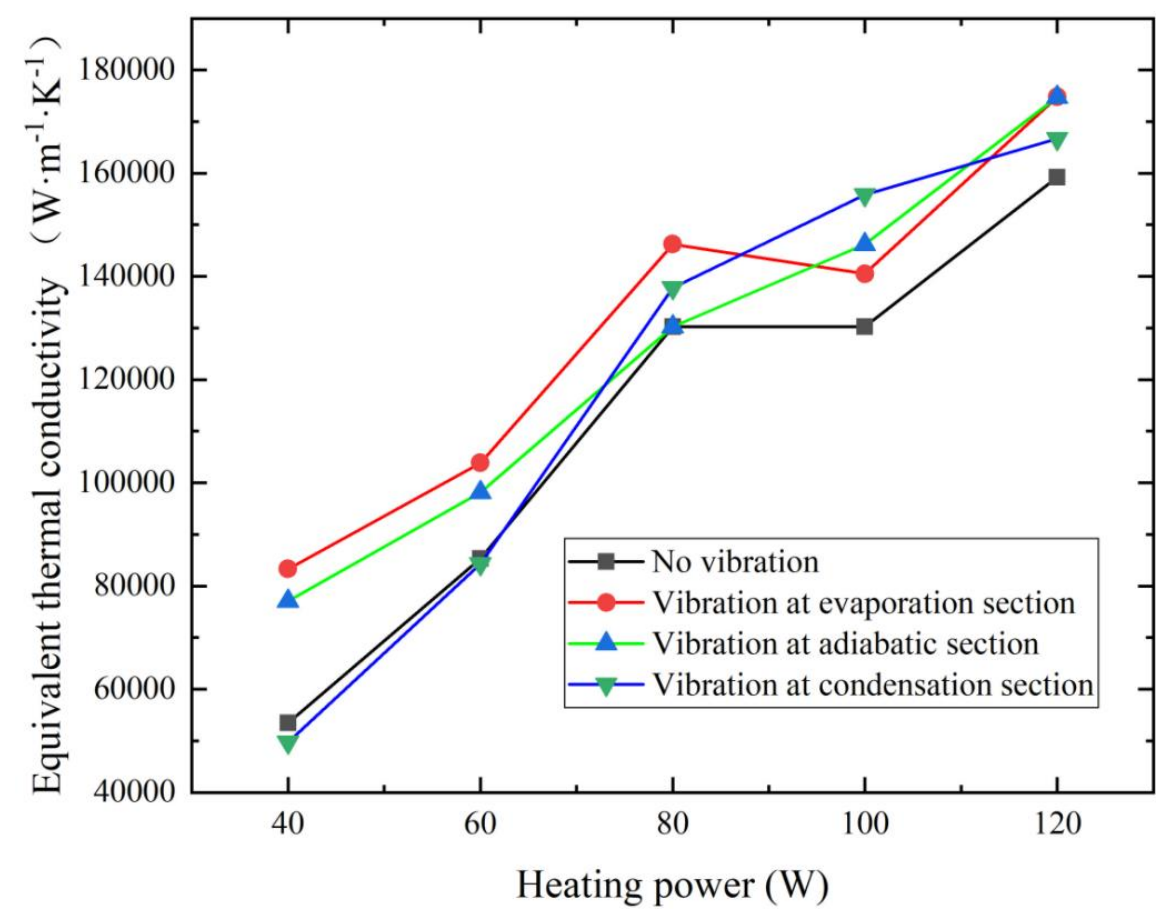

Fig. 8 Influence of vibrations of different positions on the equivalent thermal conductivity of pulsating heat pipe under different heating power levels 
The influence of vibration positions on the equivalent thermal conductivity of the pulsating heat pipe is similar to that on the thermal resistance. As shown in Fig. 8, the influences of vibrations at the evaporation section and at the adiabatic section on the equivalent thermal conductivity of the pulsating heat pipe are much more obvious than that at the condensation section. Since the equivalent thermal conductivity and the thermal resistance is reciprocal, the equivalent thermal conductivity will increase with the increase of the heating power level.

\subsection{Visualization experiments}

In order to reveal the influence mechanism of vibrations on the starting-up characteristic of the pulsating heat pipe, a high-speed camera (shooting time interval $0.1 \mathrm{~s}$ ) was used to film the evaporation section of the pulsating heat pipe, and the bubble generation and rupture states were compared under non-vibration condition and vibration condition at evaporation section, as shown in Fig. 9. It can be seen from Fig. 9 that the bubbles experience 12 pictures from generation to bursting (i.e., a total of $1.2 \mathrm{~s}$ ) under the vibration-free condition, while under the vibration condition, the bubbles experience only 6 pictures from generation to bursting (i.e., a total of $0.6 \mathrm{~s}$ ). The phenomena infer that the vibration in the evaporation section can accelerate the escape speed of the boiling bubble of the working medium, which is conducive to the start of the pulsating heat pipe.

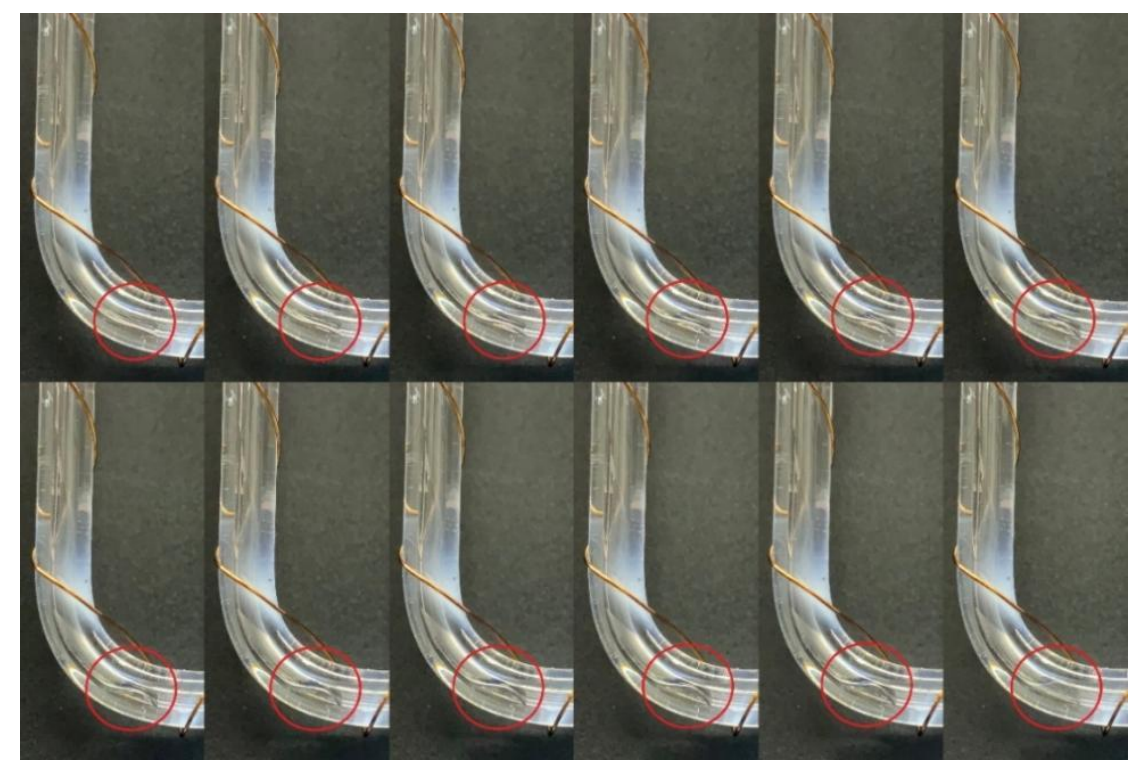

(a) 


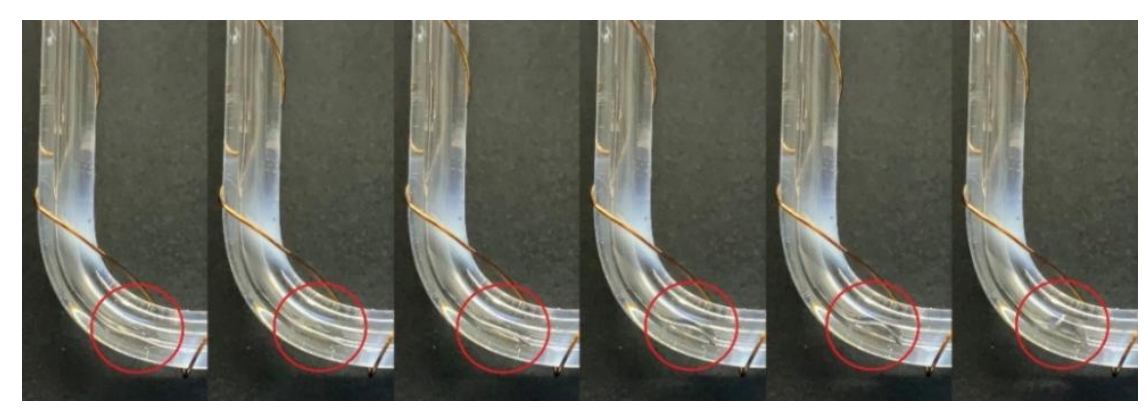

(b)

Fig.9 Dynamic photos (shooting time interval is 0.1s) of bubble generation and rupture in the evaporating section of pulsating heat pipe under

(a) vibration-free condition, and (b) vibration condition

Meanwhile, the gas plug pulsating velocity under the vibration-free condition and the vibration condition were observed by the high-speed camera as well, which is shown in Fig.10. The results in Fig.10 manifest that the gas plug pulsating velocity under the vibration condition is significantly higher than that under the vibration-free condition.

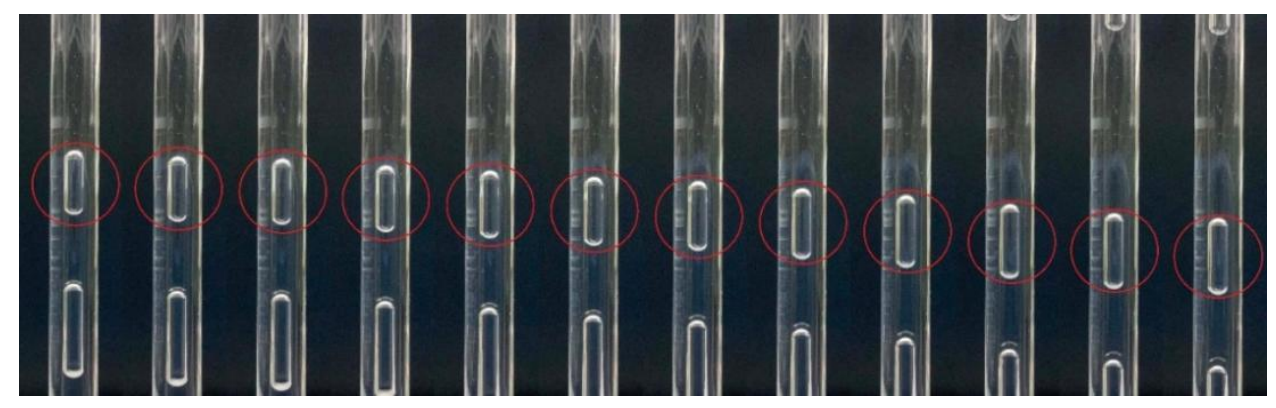

(a)

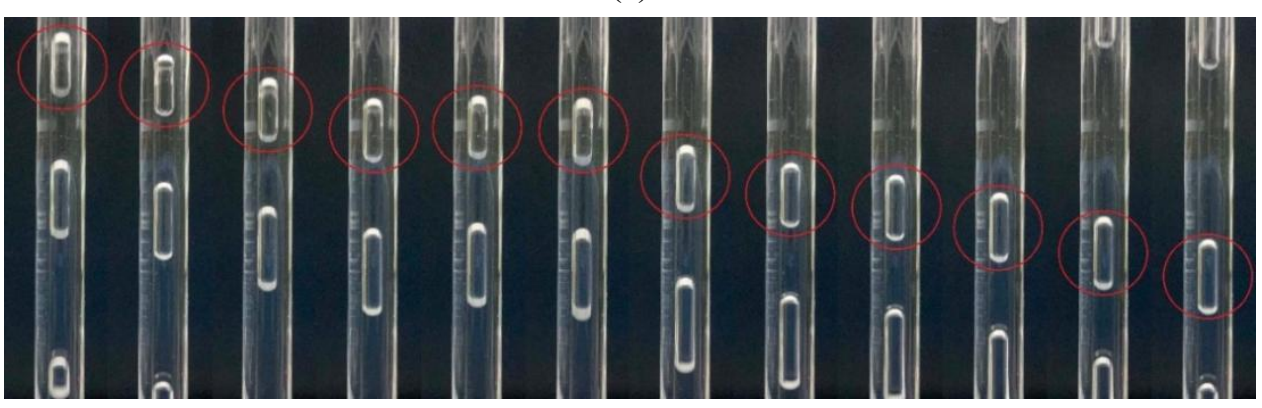

(b)

Fig.10 Dynamic photos (shooting time interval is 0.1s) of gas plug movement under (a) vibration-free condition, and (b) vibration condition

\section{Conclusions}

This study mainly experimentally investigates the influence of vibration positions on the startingup and heat transfer characteristics of pulsating heat pipe. The following conclusions can be made as 
below:

1) The starting-up time of pulsating heat pipe decreases with the increase of heating power level. The vibrations at different positions can reduce the starting-up time of pulsating heat pipe, and the smaller the heating power level is, the better the reduction effect will be. The effect of vibrations at the evaporation section and at the adiabatic section on the starting-up time of pulsating heat pipe is more significant than that at the condensation section.

2) The starting-up temperature of the pulsating heat pipe firstly decreases and then increases with the increase of the heating power level. The vibrations at different positions can reduce the startingup temperature of the pulsating heat pipe. The effect of the vibrations at the evaporation section is the best as the heating power is lower, and the effect of the vibration at the adiabatic section is the best as the heating power is higher.

3) The thermal resistance of the pulsating heat pipe decreases with the increase of the heating power level. The vibrations at the evaporation section and at the adiabatic section can reduce the thermal resistance of the pulsating heat pipe. The smaller the heating power level is, the better the reduction effect will be achieved by the vibrations at the evaporation section and at the adiabatic section. However, the vibrations at the condensation section has little effect on the thermal resistance of the pulsating heat pipe.

4) With the increase of heating power, the evaporation section temperature of pulsating heat pipe firstly increases, then basically remains unchanged, and then continues to increase. Under the lower heating power, the vibrations at the evaporation section and at the adiabatic section can effectively reduce the temperature of evaporation section of the pulsating heat pipe, but the vibrations at the condensation section has no effect on the temperature of evaporation section of the pulsating heat pipe.

\section{Acknowledge:}

This work was financially supported by Research Fund Project of Nanjing Vocational Institute of Transport Technology (Grant No. JZ1602) and National Natural Science Foundation of China (NSFC) Grant No.11874326

\section{References}

[1] D. B. Tuckerman, R. F. W. Pease. High-performance heat sinking for VLSI [J]. IEEE Electron Device Letters, 1981, 2(5): 126-129. 
[2] M. A. Ebadian, C.X. Lin . A review of high-heat-flux heat removal technologies [J]. Journal of Heat Transfer, 2011, 133(11): 110801.

[3] H. Akachi, Structure of a Heat Pipe, 1990, U.S. Patent 4921041.

[4] K. Bao, X. Wang,Y. Fang, X. Ji, X. Han, G. Chen. Effects of the surfactant solution on the performance of the pulsating heat pipe [J]. Applied Thermal Engineering,2020,178:115678

[5] A. Gandomkar, K. Kalan, M. Vandadi, M. B. Shafii, M. H. Saidi. Investigation and visualization of surfactant effect on flow pattern and performance of pulsating heat pipe [J]. Journal of Thermal Analysis and Calorimetry,2020,139(3):2099-2107

[6] C. Zhang, R.Xu, J. Chen, Q.WU. Analysis of start-up characteristics of pulsating heat pipe with zeotropic immiscible mixtures [J]. Chemical Industry and Engineering Progress, 2019, 38(12):5279-5286.

[7] H. Ahmad, S. K. Kim, S. Y. Jung. Analysis of thermally driven flow behaviors for two-turn closed-loop pulsating heat pipe in ambient conditions: An experimental approach [J]. International Journal of Heat and Mass Transfer, 2020,150(Apr.):119245.1-119245.13.

[8] L.Bai, X.Ren, W.Yang.Vacuum effect on heat transfer performance of the pulsating heat pipe [J]. Cryogenics and Superconductivity, 2019,47(04):62-66

[9] X. Huang, X. Lu, G. Xu, X. Chen, Y. Duan. Analysis of flow pattern and heat transfer characteristics of pulsating heat pipe under different thermal loads [J]. Applied Energy Technology, 2019(11):13-20.

[10] K. Bao, C. Hua, X. Wang, X. Han, G. Chen. Experimental investigation on the heat transfer performance and evaporation temperature fluctuation of a new-type metal foam multichannel heat pipe [J]. International Journal of Heat and Mass Transfer,2020,154.

[11] E. Jiang, D.Zhang, J.Zhou, S.Chao. Numerical simulation of pulsating heat pipes with twobends in different structures [J]. Journal of Chemical Industry, 2019,70(S2):244-249

[12] A. Alaei, M.H. Kafshgari, H. Atashi. A new designed heat pipe: an experimental study of the thermal performance in the presence of low-frequency vibrations[J]. Heat and Mass Transfer, 2012, 48(4): 719-723.

[13] A.Alaei, M.H. Kafshgari, S. K. Rahimi. A vertical heat pipe: an experimental and statistical study of the thermal performance in the presence of low-frequency vibrations[J]. Heat and Mass 
Transfer, 2013, 49(2): 285-290.

[14] A. Alaei, M. H. Kafshgari. Low-Frequency vibrations on the thermal performance of an oscillating heat pipe[J]. Industrial \& Engineering Chemistry Research, 2014, 53(30): 12179-12183.

[15] R.H.Chen, Y.J. Lin, C.M. Lai. The influence of horizontal longitudinal vibrations and the condensation section temperature on the heat transfer performance of a heat pipe $[\mathrm{J}]$. Heat Transfer Engineering, 2013, 34(1): 45-53.

[16] C.Guo, X. Hu, W. Cao. Effect of mechanical vibration on flow and heat transfer characteristics in rectangular microgrooves[J]. Applied Thermal Engineering, 2013, 52(2): 385-393.

[17] L.Zhang, J.Lv, M.Bai. Effect of vibration on forced convection heat transfer for SiO-Water nanofluids[J]. Heat Transfer Engineering, 2015, 36(5): 452-461

[18] S.W. Chen, T. Hibiki, M. Ishii. Experimental investigation of horizontal forced-vibration effect on air-water two-phase flow[J]. International Journal of Heat and Fluid Flow, 2017, 65: 33-46.

[19] H. Zhou. Study on flow and heat transfer performance of pulsating heat pipe under vibration [D]. Suzhou University of Science and Technology,2019. 